\title{
Erratum to: Prenatal nicotine exposure induces HPA axis-hypersensitivity in offspring rats via the intrauterine programming of up-regulation of hippocampal GAD67
}

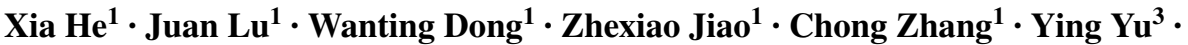 \\ Zhaohui Zhang ${ }^{3} \cdot$ Hui Wang ${ }^{1,2} \cdot$ Dan $\mathrm{Xu}^{1,2}$
}

Published online: 3 August 2017

(C) Springer-Verlag GmbH Germany 2017

\section{Erratum to: Arch Toxicol \\ DOI 10.1007/s00204-017-1996-8}

The authors of the above article would like to apologise for the mistakes which are present in Fig. $7 \mathrm{~b}$ and in Fig. 7 legend. In Fig. 7b, the immunofluorescence pictures should be corrected as below. In Fig. 7 legend, the "(nt -358 to -77)" should be corrected as "(nt -1019 to -689$)$ ".

A corrected version of this figure and figure legend are as below:

The online version of the original article can be found under doi:10.1007/s00204-017-1996-8.

Dan Xu

xuyidan70188@whu.edu.cn

1 Department of Pharmacology, Basic Medical School of Wuhan University, Wuhan 430071, China

2 Hubei Provincial Key Laboratory of Developmentally Originated Disease, Wuhan 430071, China

3 Department of Neurology, Renmin Hospital of Wuhan University, Wuhan 430060, China 

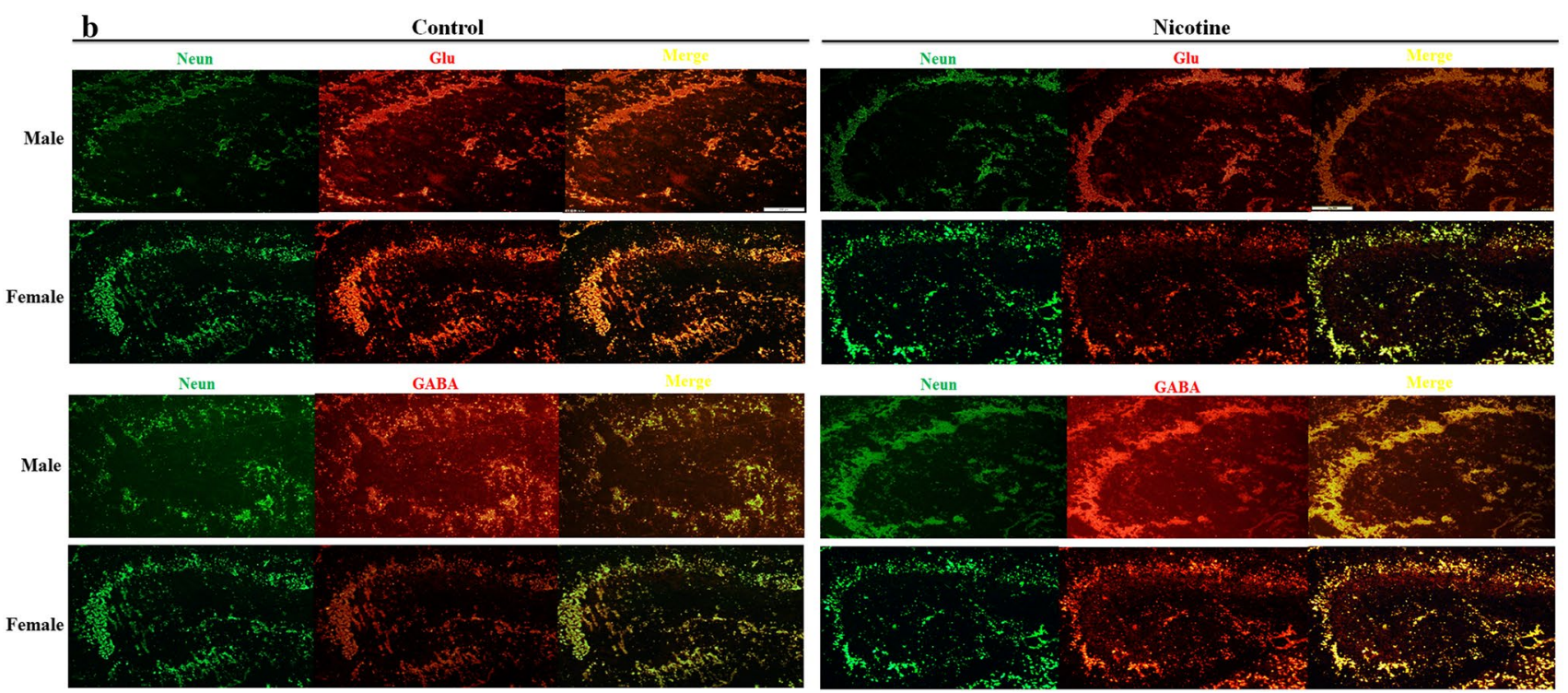

Fig. 7 Effects of prenatal nicotine exposure on the function of the hippocampus of fetal rats. Pregnant rats were subcutaneously administered $2.0 \mathrm{mg} / \mathrm{kg}$ day of nicotine from gestational day (GD) 9 to GD20, and then the fetal rats were extracted. The number of pregnant rats in each group was set to 15 (a litter size of 8 to 14 was considered qualified). The fetal hippocampus tissues were collected, and samples of each gender collected from each littermate were combined. The mRNA expression levels of glutamic acid decarboxylase 67 (GAD67), $\alpha 4$ and $\beta 2$ subtype of nicotinic acetylcholine receptor $(\alpha 4 \beta 2 \mathrm{nAChR})$ and DNA methyltransferase 1 (Dnmt1) were detected by RT-qPCR (a, d, $n=15$ per gender per group). Hippocampal neurons were detected by immunofluorescence staining, while the representative confocal laser-scanning microscopic images were double-stained for Glu (red, glutamatergic neuronal marker) or GAD67 (red, GABAergic neuronal marker) and NeuN (green, neuronal nuclei marker) $(\mathbf{b}, n=4$ per gender per group). Quantitative analysis for Glu-positive and GABA-positive cells in immunofluorescence was calculated (c). The methylation status of GAD67 promotor (nt -1019 to -689 ) was detected by bisulfitesequencing PCR method (d, $n=4$ per gender per group). Mean $\pm \mathrm{SD}$, $* P<0.05$ compared with control 